\title{
LA CONFIGURACIÓN DE LOS RIESGOS EN EL SEGURO MARÍTIMO
}

\author{
THE CONFIGURATION \\ OF RISKS IN MARITIME INSURANCE
}

\author{
GIOVANNI BERTI DE MARINIS* \\ Fecha de recepción: 28 de octubre de 2021 \\ Fecha de aceptación: 1 de diciembre de 2021 \\ Disponible en línea: 30 de diciembre de 2021
}

Para citar este artículo/To cite this article

BeRTI DE MARINIS, Giovanni. La configuración de los riesgos en el seguro marítimo, 55 Rev. Ibero-Latinoam.Seguros, 181-200 (2021). https://doi.org/10.11144/Javeriana.ris55.crsm

doi:10.11144/Javeriana.ris55.crsm

\footnotetext{
* Profesor Titular de Derecho de la economía en la Universidad de Perugia y acreditado como Catedrático en Derecho de la economía. Obtuvo el título de Doctorado en Derecho Civil en la Universidad de Camerino en la que también obtuvo el título de especialista en Derecho Civil. Forma parte de los docentes del Doctorado internacional en "Diritto dei consumi" de la Universidad de Perugia, y de los Consejos científícos de varias revistas académicas en Italia y España. Es autor de varias monografías y de numerosos escritos en temas de Derecho civil, Derecho de la competencia, Derecho de seguros y Derecho bancario. Contacto: giovanni. bertidemarinis@unipg.it
} 


\section{RESUMEN}

Este trabajo analiza las peculiaridades que surgen en la normativa sectorial específica relativa al seguro marítimo en cuanto a la identificación de los riesgos asegurados. De forma preliminar, se examinan las diferencias que se pueden encontrar entre las reglas generales del contrato de seguro contenidas en el código civil italiano y las expresamente dedicadas al seguro marítimo contenidas en el código de navegación.

La atención se centra, pues, en las cuestiones relativas a la posibilidad de asegurar riesgos putativos y de dar un carácter retroactivo al seguro marítimo. Distinguiendo, entonces, el seguro de buques del seguro de mercancías, se analizan las disposiciones específicas que regulan el tiempo de duración de la cobertura del seguro en cuestión con el fin de verificar en que circunstancias el siniestro está cubierto por la póliza. Finalmente, se examinan las cuestiones relativas al impacto en el contrato de seguro marítimo de eventos que modifiquen el riesgo asegurado.

Palabras claves: Seguro marítimo; Riesgos asegurables; Riesgos putativos; Extensión temporal de la cobertura; Modificacion en el riesgo asegurado.

\section{ABSTRACT}

This work analyzes the peculiarities that arise in the specific sectoral regulations relating to maritime insurance in terms of the identification of the insured risks. In a preliminary way, the differences that can be found between the general rules of the insurance contract contained in the Italian civil code and those expressly dedicated to maritime insurance contained in the navigation code are examined.

The focus is therefore on questions relating to the possibility of insuring putative risks and of ensuring retroactive character to marine insurance. Distinguishing, then, ship insurance from merchandise insurance, the specific provisions that regulate the duration of the insurance coverage in question are analyzed in order to verify under what circumstances the claim is covered by the policy. Finally, the issues related to the impact on the maritime insurance contract of events that modify the insured risk are examined.

Keywords: Maritime insurance; Insurable risks; Putative risks; Temporal extension of coverage; Modification in the insured risk.

SUMARIO: 1. RIESGOS EN EL SEGURO MARÍTIMO: ART. 521 CÓDIGO DE NAVEGACIÓN ITALIANO (C. NAV.). 2. LA EVOLUCIÓN HACIA RIESGOS TIPICOS Y LA INFLUENCIA DEL SISTEMA ANGLOSAJÓN EN LA DELIMITACIÓN DE RIESGOS. 3. COBERTURA DEL DENOMINADO RIESGO PUTATIVO REGULADO POR EL ART. 514 C. NAV. 4. EL RIESGO PUTATIVO EN EL CÓDIGO DE NAVEGACIÓN Y EL REGLAMENTO GENERAL DEL CONTRATO DE SEGURO DEL CÓDIGO CIVIL. 5. LA DURACIÓN DEL SEGURO DE TIEMPO MARÍTIMO. 6. CAMBIOS EN EL RIESGO ASEGURADO. 7. SIGUE: EL CAMBIO DE RUTA, VIAJE O BARCO. 8. BIBLIOGRAFÍA. 


\section{RIESGOS EN EL SEGURO MARÍTIMO: ART. 521 CÓDIGO DE NAVEGACIÓN ITALIANO (C. NAV.)}

El contrato de seguro de daños en general, como instrumento jurídico capaz de traducir las consecuencias negativas de un siniestro, gira en torno al concepto de "riesgo" por el que entendemos la incertidumbre de que acontezca un hecho perjudicial que, no siendo ni necesario ni imposible, es incierto en el momento de la celebración del contrato $^{1}$. La relevancia práctica de este elemento se refleja en el hecho de que a través de él se circunscribe el alcance de efectividad de la cobertura y que condiciona inexorablemente el destino del contrato y la relación jurídica que éste genera. La ausencia de riesgo o el hecho de que ya haya ocurrido en el momento de la celebración del contrato determina, por regla general, la nulidad del contrato (artículo 1895 del Código Civil italiano); sus variaciones de intensidad que se producen en espera de la relación de negociación, generan la necesidad de reconfigurar la relación a la luz de la nueva realidad (artículos 1896-1898 del Código Civil)². Por último, cuando varía la intensidad del riesgo, por razones obvias, variará también la prima que el asegurado debe pagar al asegurador.

La polisemia del término y los diversos significados con los que se utiliza en el sector de los seguros ${ }^{3}$-ha llevado a la doctrina a distinguir entre riesgo "extra-seguro" y riesgo asegurado ${ }^{4}$. Con el significado de riesgo "extra-seguro" se suele entender la percepción que tiene el asegurado de un determinado hecho futuro que, de producirse, tendría consecuencias negativas en su ámbito jurídico. Por riesgo asegurado, en cambio, nos referimos habitualmente a la forma en que ese riesgo extra de seguro se tiene en cuenta dentro del contrato, delimitando así el riesgo que se transfiere del asegurado al asegurador. Esta distinción, en muchos aspectos meramente descriptiva ${ }^{5}$, sirve para comprender la relación que se crea entre el riesgo asegurado y la obligación asumida por el asegurador que será llamado a indemnizar el asegurado por la ocurrencia del riesgo solo si -y en la medida en que-el mismo se haya deducido dentro del contrato de seguro.

El punto parece ser central, precisamente en relación con la disciplina del riesgo en el seguro marítimo que, no obstante estar sujetos a las regulaciones generales del Código Civil gracias a lo que dispone el art. 1885 del Código Civil italiano, se ven afectados

\footnotetext{
${ }^{1}$ Cfr., Ferrari. Il problema dell'alea contrattuale, Napoli, 2001, p. 100; RossetTI. Il diritto della assicurazioni, I, Padova, 2011, p. 751; CoRRIAS. Il problema dell'onerosità-gratuità nel contratto di assicurazione e nei contratti aleatori, in Riv. dir. civ., 2016, p. 68; Mazzola. Sul concetto di interesse nel contratto di assicurazione: inquadramento teorico e profili applicativi, ivi, 2019, p. 1201 ss.

2 Giampaolino. Le assicurazioni, in Tratt. dir. comm. Buonocore, Torino, 2013, pp. 182 ss.

3 Reconstruye los significados multiformes con los que se usa el término, DonATI, Trattato del diritto delle assicurazioni, vol. II, Milano, 1954, pp. 111 ss.

${ }^{4}$ Fanelli. Le assicurazioni, in Tratt. dir. civ. comm. Cicu e Messineo, Milano, 1973, pp. 101 ss.

5 Critican la utilidad de esta distinción, entre otros, ANGELICI. Aggravamento e modifiche del rischio, in Assicurazioni, 1985, I, p. 549; DonATI. Trattato del diritto delle assicurazioni, cit., p. 113 ss.; RosSETTI. Il diritto delle assicurazioni, cit., p. 752.
} 
también por las disposiciones específicas contenidas en el Código de Navegación en los artículos 514 y siguientes ${ }^{6}$.

Los seguros marítimos, en su distinción en pólizas de buques, de bienes y de responsabilidad, pertenecen a la familia de pólizas de seguros de daños que, en el contexto del código civil, tienden a dejar a las partes la posibilidad de identificar en el mismo contrato los riesgos sujetos a coberturas sin que sean predeterminados por el legislador. La disciplina especial, sin embargo, introduce en el art. $521 \mathrm{c}$. nav., una primera distinción relevante al tipificar los riesgos cubiertos por el seguro marítimo ${ }^{7}$. El artículo, en la interpretación reiterada de la doctrina, lleva a afirmar que el seguro marítimo es un contrato que cubre una universalidad de riesgos ${ }^{8}$ que incluyen daños y perjuicios que afecten a los bienes asegurados por tormentas, naufragios, choques, explosiones, incendios, piratería, saqueos y en general por todos los accidentes de navegación ${ }^{9}$.

Si bien el artículo mencionado lleva como rúbrica "Riesgos de la navegación", no ha escapado a la doctrina de que muchos de los eventos negativos deducidos en el mismo pueden calificarse como siniestros que generan necesariamente "daños y pérdidas" (p. Ej., Naufragio, incendio, explosión, impacto, etc.), más que como riesgos reales que pretenden exponer al asegurado a un peligro que, al producirse, podría causar daños ${ }^{10}$. Sin embargo, cabe señalar que el listado al que nos referimos es meramente un ejemplo, encontrando, entonces, su unidad en la cláusula general que cierra el art. 521 c. nav. declarando que "todos los accidentes de navegación" están cubiertos ${ }^{11}$.

Esta cláusula general se interpreta en el sentido de incluir cualquier riesgo que pueda afectar al buque o las mercancías transportadas por mar y, por tanto, tiende a incluir tanto los riesgos que provienen del mar como los que se derivan directamente del buque $^{12}$. La amplitud de los riesgos incluidos en la cobertura de conformidad con el art. 521 c. nav. ${ }^{13}$, empero, se atenúa por la necesidad de que los hechos que causalmente

\footnotetext{
${ }^{6}$ Sobre la especialidad de las disposiciones del Código de la Navegación respecto a las que, en el Código Civil, rigen los seguros, v. FerRarinI. Le assicurazioni marittime, Milano, 1990, p. 9 y, en la jurisprudencia, Cass., 21 julio 1962, n. 1989, in Foro it., 1962, I, c. 1900.

7 De manera preliminar, sobre este punto,GRECHI, Brevi considerazioni sul concetto di rischio nelle assicurazioni marittime, in Dir. prat. ass., 1962, pp. 184 ss.

${ }^{8}$ Ferrarini. Le assicurazioni marittime, cit., p. 111; ID., comentario a Cass., 6 febbraio 1953, n. 302, in Assicurazioni, 1953, II, p. 175; Querci, Diritto della navigazione, Padova, 1989, p. 609.

9 V., Riccardelli. Lezione di diritto della navigazione: assicurazioni marittime, in Dir. trasp., 2017, p. 940, que destaca que "el seguro marítimo es un seguro contra una universalidad de riesgos, mientras que en otros seguros distintos de los de vida el riesgo, por regla general, debe ser identificado positivamente, es decir, el asegurador garantiza solo ciertos riesgos indicados por su nombre".

10 En este sentido, Righettr. Trattato di diritto marittimo, III, Milano, 1994, p. 614; BALESTRA. Le assicurazioni marittime dei rischi di guerra, Milano, 1991, p. 20.

11 RicCARDelli. Lezione di diritto della navigazione: assicurazioni marittime, cit., p. 941.

12 Ferrarini. Le assicurazioni marittime, cit., p. 110; Lefebvre D’Ovidio, Pescatore e Tullio. Manuale di diritto della navigazione, $9^{\mathrm{a}}$, Milano, 2000, pp. 714 ss.

13 V., sobre el punto, Di Marco. La funzione del contratto di assicurazione marittima, in Assicurazioni, 2004, p. 411, quien incluye en los riesgos de navegación, "todos aquellos eventos, propios del entorno en el que se desarrolla la navegación, que puedan ocasionar daños o extravíos de los elementos asegurados; los efectos (impacto en el asegurador) son equiparados a estos riesgos por los eventos, que pueden no ser
} 
determinan el accidente sean de carácter extraordinario, no cubriendo así aquellos "peligros del mar" que ocurren por la acción ordinaria de las aguas y los vientos ${ }^{14}$.

Este enfoque adoptado por nuestro Código de Navegación, determina la consecuencia práctica de toda importancia por la cual, a fin de excluir o limitar la incidencia de ciertos riesgos sobre la prestación prometida por el asegurador deberá excluir de forma expresa, mediante un convenio contractual adecuado, la cobertura de eventos que puedan relacionarse de manera abstracta con un "accidente de navegación"15. Esto, como se mencionó anteriormente, marca una diferencia sustancial entre el seguro marítimo y el seguro común en el que, por el contrario, el alcance de la cobertura se suele identificar enumerando los riesgos incluidos ${ }^{16}$.

\section{LA EVOLUCIÓN HACIA RIESGOS TÍPICOS Y LA INFLUENCIA DEL SISTEMA ANGLOSAJÓN EN LA DELIMITACIÓN DE RIESGOS}

A pesar de los datos textuales, la práctica del seguro en el sector marítimo muestra una desviación sustancial del enfoque asumido por el Código de Navegación. Esta evolución está de hecho determinada por la influencia que los esquemas contractuales anglosajones han asumido también en las negociaciones internas ${ }^{17}$. El sistema inglés, a diferencia del nacional (italiano), parece estar orientado a cubrir únicamente los riesgos mencionados, no incluyendo la cobertura universal de los "Riesgos de la navegación" como afirma, al contrario, el art. $521 \mathrm{c}$. nav. ${ }^{18}$. Como resultado de esta característica, el Instutute of London Underwriter ha elaborado cláusulas específicas que, conteniendo un listado completo de los riesgos incluidos y los riesgos excluidos, determinan

propios del entorno en el que se desarrolla la navegación, pero respecto de los cuales se reflejan las peculiaridades de este entorno, determinando un cambio y / o agravamiento del riesgo".

14 RicCARdelli. Lezione di diritto della navigazione: assicurazioni marittime, cit., p. 941, quien destaca cómo los peligros del mar "no incluyen la acción ordinaria de las aguas y los vientos, porque el barco debe estar en condiciones tales que resista la acción ordinaria de las olas; en cambio, la tormenta se encuentra entre los peligros del mar. En definitiva, los peligros de mar se refieren a hechos anormales que pueden perturbar la navegación, ya que si el accidente se produce por la acción normal de las aguas, el buque asegurado evidentemente presentaba un defecto oculto y no estaba apto para navegar". Sobre el punto, ID., Il vizio occulto nell'assicurazione della nave, Padova, 1970, pp. 12 ss.

15 Di Marco. La funzione del contratto di assicurazione marittima, cit., pp. 411 ss. donde también destaca las diferencias entre la expresión "accidentes de navegación” utilizada por el código de navegación y la de «perils of the seas» de la ley anglosajona que incluye sólo los riesgos "del" mar (of the seas) y no los peligros que pueden producirse en los mares (on the seas) y, por tanto, quedan "excluidos aquellos riesgos que se producen en el mar, sin que por ello sean producidos por él, y que por el contrario entran dentro de nuestra acepción de accidentes de navegación”. Ver también, QuERCI. Diritto della navigazione, cit., p. 610. 16 RicCARDELl. Lezione di diritto della navigazione: assicurazioni marittime, cit., p. 941, donde destaca que la expresión "todos los accidentes de navegación", de hecho, "invierte la relación entre los riesgos cubiertos y los riesgos excluidos con respecto al seguro terrestre, ya que la lista de riesgos excluidos es obligatoria; por tanto, se debe contemplar expresamente un riesgo de exclusión".

17 Así Ferrarini. Le assicurazioni marittime, cit., pp. 117 ss.

18 Di Marco. La funzione del contratto di assicurazione marittima, cit., p. 412, habla de «named perils» en relación con los riesgos cubiertos por las pólizas anglosajonas. 
negocialmente el alcance de la cobertura. Tales modelos ${ }^{19}$ se utilizan con frecuencia o se mencionan en los contratos nacionales, lo que genera una contaminación sustancial entre los sistemas legales que, como se mencionó anteriormente, tienen enfoques muy diferentes sobre el tema. Esto ha llevado a parte de la doctrina a considerar obsoleto el concepto de universalidad de los riesgos asegurados que surge del art. 521 c. nav., precisamente por los tecnicismos típicos de las especificaciones en uso en el sector asegurador que, de hecho, han llevado a una tipificación de los riesgos cubiertos por el seguro ${ }^{20}$.

El fenómeno del uso de formulas contractuales extranjeras en los contratos de seguro italianos produce, además de las consecuencias generales descritas anteriormente, también problemas de carácter práctico. El carácter anfibio de estos contratos, de hecho, plantea ante todo dudas sobre la identificación de la ley aplicable al contrato $\mathrm{y}$, en concreto, si el mismo contrato se rige por disposiciones italianas o extranjeras. Sobre este perfil, precisamente para evitar las incertidumbres debidas a la identificación a través de los criterios de conexión propios del derecho internacional privado de la ley aplicable al contrato de seguro ${ }^{21}$, las pólizas italianas identifican negocialmente la ley aplicable al contrato -incluyendo las cláusulas extranjeras a las que se hace referencia-, como regla general en la ley italiana ${ }^{22}$.

Otro problema al que se enfrenta la doctrina se refiere a la interpretación de estos contratos con especial referencia a las cláusulas extranjeras mencionadas. En estos contratos, de hecho, a los problemas específicos relacionados con la interpretación del contrato de seguro $^{23}$, se suma la incertidumbre adicional que deriva del hecho de utilizar un lenguaje técnico/legal que, a veces, se aleja del continental ${ }^{24}$. La necesidad de uniformidad derivada principalmente del alcance transfronterizo de los contratos de seguro marítimo, han llevado a la doctrina mayoritaria a considerar oportuno interpretar las cláusulas de los modelos contractuales anglosajones según el significado que tendrían en el mercado de origen ${ }^{25}$.

\footnotetext{
19 Es el Institute Time Clauses- Hulls (ITCH) del 1983 para el seguro de buques y el Institute Cargo Clauses (I.C.C.) para los seguros de mercancías. Véase a Siccardi. Le nuove Institute Time Clauses - Hull, in Dir. mar., 1984, pp. 975 ss; QuerCI. Diritto della navigazione, cit., pp. 608 ss; SAlEmI. Le assicurazioni marittime e trasporti, in CANDIAN e PACI (a cura di) Manuale di tecnica delle assicurazioni, Milano, 2002, pp. $541 \mathrm{ss}$.

${ }^{20}$ Così, FERrarini. Le assicurazioni marittime, cit., p. 111; RighetTI. Trattato di diritto marittimo, cit., p. 613.

${ }^{21}$ Cfr., con referencia al contrato de seguro en general, Heiss. Insurance Contracts in Rome I: Another Recent Failure of the European Legislature, in Yearbook of Private Int. Law, 2008, 261 ss.; PretelLI. Il regolamento comunitario sulla legge applicabile alle obbligazioni contrattuali (Roma I), in Eur. dir. priv., 2009, pp. 1083 ss.

22 Di Marco. La funzione del contratto di assicurazione marittima, cit., pp. 415 ss., quien pone de relieve esta tendencia tanto en las pólizas de buques como en las de mercancías.

23 V., Rossetti. Il diritto della assicurazioni, I, cit., pp. 1033 ss.

${ }^{24}$ Cfr., preliminarmente, MoliterNI. La clausola “da magazzino a magazzino” nell'assicurazione marittima delle merci e l'interpretazione del contratto, in Nuova giur. civ. comm., 2005, pp. 209 ss.

25 Así Ferrarini. Le assicurazioni marittime, cit., p. 29; ID., Polizze tipo italiane e clausolari inglesi di assicurazioni marittime, in Assicurazioni, 1981, I, p.125. Tal tesis es seguida por Piombino. L'impiego di clausolari stranieri nel contratto di assicurazione marittima: la soluzione adottata dalla nuova polizza corpi dell'ANIA, in Dir. mar, 1989, p. 1216 ; Di MARCo. La funzione del contratto di assicurazione
} 


\section{COBERTURA DEL DENOMINADO RIESGO PUTATIVO REGULADO POR EL ART. 514 C. NAV.}

Un rasgo ciertamente peculiar del seguro marítimo se encuentra en la disciplina específica reservada a los llamados riesgos putativos contenida en el art. 514 c. nav. En general, la cobertura del riesgo putativo significa la posibilidad de que el asegurador sea responsable de un siniestro que ya se ha producido con antelación al momento de la celebración del contrato, pero que las partes aún no conocen.

El origen de los llamados riesgos putativos se encuentra en el art. 430 del Código de Comercio de 1882 - ya no vigente-que sancionaba efectivamente la nulidad del contrato de seguro si, al momento de su celebración, “el asegurador y el asegurado o la persona que contrató el seguro conocían la falta o extinción de los riesgos o la ocurrencia del daño"26. Entendido en este sentido, los riesgos potencialmente sujetos a cobertura serían también todos aquellos cuya incertidumbre es apreciable solo a nivel subjetivo de las partes que desconocen que el riesgo se haya ya producido ${ }^{27}$. Sin embargo, este enfoque cambia radicalmente con la unificación de los dos códigos (civil y mercantil) en el código civil de 1942 que, integrando las reglas del contrato de seguro en los artículos 1882 ss. del Código Civil italiano, con el art. 1895 del mismo Código Civil, afirma la nulidad del contrato de seguro "si el riesgo nunca existió o dejó de existir antes de la celebración del contrato". Con este cambio de posición, por lo tanto, la orientación dominante reafirma que el riesgo putativo no puede cubrirse mediante contratos de derecho común y que permanece vivo, precisamente como resultado del art. 514 c. nav., solo en los seguros marítimos ${ }^{28}$.

La necesidad de una disposición específica que garantice, dentro de los límites que se destacarán a continuación, la posibilidad de que el asegurador sea responsable de los daños derivados de riesgos que ya no son actuales o de accidentes ya ocurridos en el momento de la estipulación del contrato, encuentra su origen en las peculiaridades que caracterizan la actividad específica del comercio marítimo. El Código de Navegación, de hecho, ve la luz en un momento histórico en el que, a pesar de los avances tecnológicos en las telecomunicaciones, aún era posible hipotetizar un desfase de tiempo entre la ocurrencia de un "accidente de navegación" y el momento en que la noticia del citado evento llega a los interesados ${ }^{29}$. A pesar del hecho que no

marittima, cit., p. 417, según el cual "la interpretación de las cláusulas inglesas, por tanto, debe llevarse a cabo en relación con el significado que la ley inglesa les atribuye. Esto significa, teniendo en cuenta el sistema de common law, que la interpretación no solo debe ser la del mercado inglés, sino también la que da la jurisprudencia británica". Para una reconstrucción diferente, sin embargo, App. Milano, 9 junio 1972, in Assicurazioni, 1973, II, 2, p. 266, comentada por GRIGOLI, Sulla delimitazione del rischio di guerra nell'assicurazione marittima.

26 Vease, Vernizzi. Il rischio putativo, Milano, 2010, pp. 75 ss.

27 En relación al riesgo, distingue entre incertidumbre objetiva e incertidumbre subjetiva GAMBINO. L'assicurazione nella teoria dei contratti aleatori, Milano, 1964, p. 72.

28 VERNIZZI. Rischio putativo ed assicurazioni retroattive tra diritto della navigazione e diritto comune, in Riv. dir. nav., 2016, pp. 219 ss.

29 Cfr., Riccardelli. Lezione di diritto della navigazione: assicurazioni marittime, cit., p. 940 quien destaca cómo surge esta preocupación en el mismo informe ilustrativo al código de navegación. 
se pueda excluir del todo que esta eventualidad también pueda ocurrir hoy ${ }^{30}$, parece sin embargo evidente que el alcance práctico de la disposición analizada es, también para las observaciones que se harán en breve, muy limitado por la inmediatez con que ahora se puede hacer circular la noticia del accidente o de la inexistencia o de la cesación del riesgo debito a la eficiencia de los medios de comunicación actuales ${ }^{31}$.

Queriendo profundizar en el tema, no se pueden dejar de notar las profundas diferencias que hay entre el art. 514 c. nav., y la disciplina contenida en el Código Civil y la que, anteriormente, estaba contenida en el art. 430 del ya derogado Código de Comercio de $1882^{32}$.

En primer lugar, cabe señalar que si bien el Código de Comercio de 1882 hacía depender la vigencia del contrato de un elemento subjetivo -como el conocimiento personal efectivo de la inexistencia del riesgo asegurado- la regulación actual del Código de Navegación se refiere a un supuesto objetivo ${ }^{33}$. La ley, en efecto, afirma la nulidad del contrato en el caso de que la noticia de la actualidad del riesgo o del siniestro haya llegado en el lugar de estipulación -o en el lugar desde donde el asegurado dio la orden de asegurar- antes de la estipulación del contrato ${ }^{34}$.

Este dato ha llevado a la doctrina a dudar del hecho de que el art. 514 c. nav., a pesar de la rúbrica que lo acompaña, concreta en realidad una hipótesis de riesgo putativo. Bajo este perfil, se observa de hecho una convergencia sustancial de las disposiciones hacia una evaluación objetiva de la presencia de un riesgo que, en el art. 514 c. nav., está anclado a la llegada objetiva de la noticia de la posible terminación del riesgo o de la ocurrencia del siniestro independientemente del estado subjetivo de los contratantes ${ }^{35}$.

30 Cfr., Grigoli. In merito alla disciplina dell'assicurazione del rischio putativo, in Assicurazioni, 1978, p. 620 il quale fa l'esempio dell'assicurazione di merce in viaggio. Nello stesso senso, CARBone, CELlE e Lopez De Gonzalo. Il diritto marittimo attraverso i casi e le clausole contrattuali, $5^{\mathrm{a}}$ ed, Torino, 2015, p. 397.

31 Considera el art. 514 c. nav. una disposición obsoleta y, de hecho, desprovista de toda justificación en la realidad moderna del tráfico marítimo, De Gregorio, in De Gregorio e Fanelli. Il contratto di assicurazione, II, Milano, 1987, p. 80.

32 Ver, sin embargo, la opinión de Querci, Diritto della navigazione, cit., p. 639, según el cual el derecho de navegación “- en cumplimiento de necesidades específicas, vinculadas a las modalidades de las relaciones y el escenario mundial, en el que se llevan a cabo-ha decidido mantener, con ligeras modificaciones, el principio que el art. 430 del Código de Comercio había fijado como norma general y que el actual Código Civil (art. 1885, cit.) había abandonado, ya que ya no correspondía a la realidad".

33 Cfr., Lefebvre D’Ovidio, Pescatore e Tullio. Manuale di diritto della navigazione, cit., p. 715; Di MARCO. La funzione del contratto di assicurazione marittima, cit., p. 419 s.

34 Sobre la identificación de lo que se entiende por lugar de estipulación y el lugar desde el cual el asegurado dio la orden de asegurar, v. Vernizzi. Il rischio putativo, cit., p. 142 s., el que destaca la necesidad de dar una interpretación amplia en el sentido de "lugares" como indicativos de "una plaza" (por ejemplo, la ciudad o el puerto de Trieste, la ciudad o el puerto de Génova)».

35 Así, Corrias. Sulle peculiarità della disciplina del rischio nelle assicurazioni marittime, in Dir. trasp., 1995, p. 51 s., donde señala que "la elección de un supuesto de estas características reafirma sustancialmente el principio establecido por el art. $1895 \mathrm{cc}$, con una excepción muy limitada justificada por la necesidad práctica, persistente a pesar del desarrollo de las telecomunicaciones, de permitir la estipulación de un contrato de seguro válido en la hipótesis excepcional en la que es presuntamente imposible que las partes contratantes conozcan el destino de los bienes en navegación. De esta regla se puede inferir, por tanto, que 
Además, desde un punto de vista práctico, no se puede ignorar que los márgenes de aplicabilidad ya existentes de la disposición están aún más restringidos como resultado del segundo párrafo del art. 514 c. nav., en virtud de lo cual se presume, mientras no se demuestre lo contrario, que la noticia ha llegado en el lugar de estipulación o en aquél desde el cual se dio la orden para asegurar una vez que ocurrió el siniestro ${ }^{36}$. Esta presunción carga al asegurado que pretende sustentar la vigencia del contrato de seguro estipulado cuando el riesgo ya se había producido, de la carga de superar la presunción antes mencionada ${ }^{37}$.

\section{EL RIESGO PUTATIVO EN EL CÓDIGO DE NAVEGACIÓN Y EL REGLAMENTO GENERAL DEL CONTRATO DE SEGURO DEL CÓDIGO CIVIL}

Si ya en el párrafo anterior era posible mencionar la diferente conformación del riesgo en los contratos de seguro regidos por el Código de Navegación en comparación con los regidos por Código Civil, ahora es necesario investigar cómo la distinta disciplina del riesgo impacta realmente en los primeros.

El primer punto a abordar se refiere a la relación que existe, en general, entre el seguro de riesgo putativo y el seguro retroactivo de la creencia generalizada, también destacada por la doctrina autorizada, según la cual el primero no sería más que una hipótesis de retroactividad de los efectos del seguro ${ }^{38}$.

En primer lugar, es necesario destacar que cuando hablamos de seguro retroactivo nos referimos a un fenómeno contractual a la luz del cual, por voluntad pactada de las partes, algunos de los efectos jurídicos producidos por el contrato se producen en un momento previo a la conclusión del mismo. Cuando, por el contrario, nos referimos a un seguro de riesgo putativo, nos referimos a hipótesis que, si bien en algunas circunstancias pueden alcanzar el mismo resultado, están, sin embargo, expresamente previstas y regidas por la ley que, como en el ahora derogado art. 430 del Código de Comercio de 1882, hace que la presencia de un riesgo asegurable dependa de un elemento subjetivo (conocimiento de las partes sobre la actualidad del riesgo) ${ }^{39}$.

Como es sabido, la posibilidad de dar efecto retroactivo a las coberturas de los seguros ha estado recientemente en el centro de un fuerte debate doctrinal y jurisprudencial centrado en la vigencia de la denominadas Cláusulas claims made dentro de los

el Código de Navegación (además del Código Civil) cambia radicalmente el régimen de riesgo establecido por el art. 430 del código comercial de 1882".

${ }_{36}$ Destaca la relevancia marginal que tiene hoy en día el arte. 514 c. nav.QuERCI. Diritto della navigazione, cit., p. 640.

37 V., Persico. Le assicurazioni marittime, Genova, 1947, p. 46 ss.; LefEbvre D’Ovidio, Pescatore e Tullio. Manuale di diritto della navigazione, cit., p. 715.

38 Ampliamente, DonATI. Trattato di diritto delle assicurazioni, cit., pp. 117 ss; GreCHI, Brevi considerazioni sul concetto di rischio nelle assicurazioni marittime, cit., pp. 184 ss.

39 Así, Volpe Putzolu. Le assicurazioni. Produzione e distribuzione. Problemi giuridici, Bologna, 1992, p. 64; Corrias. Sulle peculiarità della disciplina del rischio nelle assicurazioni marittime, cit., pp. 43 ss. 
contratos de seguro de responsabilidad civil (especialmente profesional) ${ }^{40}$. El tema, por razones obvias, no se puede explorar aquí, pero sus características destacadas pueden ayudar a comprender la relación entre la disciplina del art. 514 c. nav. y los enfoques más modernos de la retroactividad de la cobertura de seguros.

Con las precauciones que exige la jurisprudencia ${ }^{41}$, se tiende a admitir que a través de un contrato de seguro de responsabilidad civil sea posible cubrir siniestros ya ocurridos antes de la celebración del contrato pero en cumplimiento formal de lo dispuesto en el art. 1895 del Código Civil italiano. Esto ocurre en todos los casos en los que el siniestro determina la aparición del daño solo después de algún tiempo desde su ocurrencia lo que mantiene vivo un "riesgo" asegurable en todas las circunstancias en las que el daño surja por primera vez "durante el período del seguro". En tales circunstancias, persiste un riesgo ya que la ocurrencia del accidente no genera inmediatamente un daño que permanece incierto ${ }^{42}$.

En este punto es fácil detectar la relación que existe entre el art. $514 \mathrm{c}$. nav. y el art. 1895 del Código Civil italiano ya que, en primer lugar, el Código de Navegación admite -contrariamente al Código Civil y aunque con los límites señalados en el párrafo anterior- la vigencia de un seguro que cubra riesgos inexistentes, que dejaron de existir en el momento de la estipulación o que cubra siniestros que ya se han producido siempre que la noticia relativa no llegara puntualmente al lugar de estipulación ni al lugar desde donde se dio la orden de asegurar. En segundo lugar, sin embargo, la disciplina a que se refiere el art. 514 c. nav. contrasta fuertemente con las cláusulas que, de manera similar a lo que ocurre en los seguros de derecho común, prevén contractualmente su retroacción. La disposición antes mencionada, establece la nulidad del contrato incluso "si el accidente ocurrió antes de la celebración del contrato" siempre que su noticia no se reciba con prontitud en el lugar de la celebración del contrato ${ }^{43}$. En este sentido, aunque admitiendo la

\footnotetext{
40 Sobre este punto, conviene recordar por lo menos dos importantes fallos del Pleno del Tribunal supremo italiano: Cass., Sez. un., 6 mayo 2016, n. 9140, in Giur. comm., 2017, p. 983; Cass., Sez. un., 24 septiembre 2018, n. 22437, in Resp. civ. prev., 2019, p. 163. Sobre este punto, la doctrina es interminable y, en consonancia con las necesidades del presente trabajo, puede verse para todos los efecto a MugaVEro. Ancora dubbi di legittimità sulle clausole "claims made": la parola di nuovo alle Sezioni Unite, in Giur. comm., 2018, p. 501 ss.; Corrias, La clausola “claims made”" al vaglio delle Sezioni Unite: un 'analisi a tutto campo, in Banca borsa tit. cred., 2018, II, p. 656 ss.; ID., L'assicurabilità dei fatti pregressi nelle assicurazioni della responsabilità civile, in Riv. it. Med. legale, 2019, pp. 1443 ss.

${ }^{41} \mathrm{Si}$ bien la primera de las sentencias mencionadas en la nota anterior recordó al intérprete la necesidad de evaluar caso por caso los efectos concretos de estas cláusulas, la segunda de las sentencias del Pleno, considerando que las cláusulas claims made son calificables como típicas, requiere verificar caso por caso si no alteran la causa concreta del contrato.

42 Sobre este perfil, precisamente en relación al art. 514 c. nav.,CORRIAS. Sulle peculiarità della disciplina del rischio nelle assicurazioni marittime, cit., p. $47 \mathrm{~s}$.

${ }^{43}$ Cfr., otra vez, Corrias, op. cit., p. 52 ss. Contra, Ferrarini. Le assicurazioni marittime, cit., p. 115; Querci. Diritto della navigazione, cit., p. 640; Manca, Studi di diritto della navigazione, Milano, 1961, p. 271 quienes, por el contrario, parecen considerar admisible que las partes den retroactividad a la póliza siempre que el siniestro se produzca dentro del plazo cubierto por el seguro. Esta posición, sin embargo, no resulta absolutamente acorde con lo dispuesto en el art. 514 c. nav. que, en el caso de que el siniestro ya se haya producido en el momento de la celebración del contrato, sanciona la nulidad del seguro, salvo en el caso de que la noticia del siniestro aún no haya llegado al lugar de estipulación. En jurisprudencia, v.
} 
posibilidad de que se desarrollen daños a largo plazo en el sector marítimo como consecuencia de la ocurrencia de los riesgos asegurados -que, por tanto, a pesar de la ocurrencia del siniestro todavía dejan intacto un riesgo asegurable-, de hecho, para la validez del seguro de este tipo, se requiere que el accidente aún no haya ocurrido en el momento de la celebración del contrato.

No obstante, cabe señalar que, según otra doctrina, lo dispuesto en el art. 514 c. nav. sólo serviría para limitar-y no eliminar-la posibilidad de dar efecto retroactivo a las pólizas de seguros que, por tanto, serían válidas con independencia de que el siniestro ya se haya producido en el momento de la celebración del contrato, siempre que la noticia aún no haya llegado al lugar de estipulación. Sin embargo, debe objetarse la posición mencionada que, en la hipótesis propuesta por la misma - es decir: contrato estipulado cuando aún no se ha recibido la noticia del accidente-, el accidente-aunque ya haya ocurrido- sería ya cubierto por el seguro precisamente de conformidad con el art. 514 c. nav., independientemente de cualquier acuerdo que prevea la retroactividad de la póliza.

\section{LA DURACIÓN DEL SEGURO DE TIEMPO MARÍTIMO}

En parte relacionado con la problemática referida en los párrafos anteriores, surge el aspecto relativo a la duración del seguro marítimo lo que, desde un punto de vista temporal, ayuda a comprender los términos de efectividad de las pólizas. Desde este punto de vista, el Código de Navegación distingue el seguro de buques del seguro de mercancías.

En cuanto al seguro del buque, éste puede tener una duración temporal (art. 530 del Código Naval) o por viaje (art. 531 del Código Naval). La elección de una u otra fórmula depende obviamente de las necesidades específicas del asegurado que, si quiere asegurar el barco para una serie de viajes, puede vincular la duración del seguro no al viaje único sino a un plazo establecido en el contrato ${ }^{44}$.

En el caso de seguro de buque a tiempo, en total armonía con las disposiciones generales del art. 1899 del Código Civil, el mismo surtirá efectos desde la media noche del día de su celebración hasta la media noche del día señalado en la póliza, refiriéndose, en cuanto al horario, al vigente en el lugar de celebración del contrato. El segundo párrafo del art. 530 c. nav., establece una extensión automática de cobertura, previo pago de un suplemento de prima proporcional, en caso de que la póliza deje de ser efectiva mientras el buque aún se encuentre navegando. En este caso, la extensión

Cass., 24 abril 1961, n. 919, in Foro it., 1961, I, c. 1346, con comentario de CAPOTOSTI, Rischio putativo nell'assicurazione marittima di trasporto merci e nullità del contratto per avaria particolare preesistente all'imbarco.

44 Lefebvre D’Ovidio, Pescatore e Tullio. Manuale di diritto della navigazione, cit., p. 721 donde, con referencia a los seguros basados en el tiempo, destacan que "se evita así celebrar un contrato para cada viaje cuando el buque se dedica a un servicio de línea, o, si se fleta ocasionalmente, cuando tiene que ir a uno o a otro puerto para cargar a pedido del fletador". 
automática extiende la cobertura hasta la medianoche del día en que el barco llega al puerto de destino ${ }^{45}$.

Sin embargo, en el caso de que se contrate un seguro de barco de viaje, el art. 531 c. nav., establece que el mismo es efectivo desde el momento en que se inicia el embarque de las mercancías hasta el momento en que las mismas son desembarcadas en el puerto de destino del viaje, siempre que esto ocurra a más tardar a los veinte días desde el atraque y salvo lo dispuesto en el segundo párrafo del art. 531 c. nav. ${ }^{46}$. En el caso de que no se prevea el embarque de mercancías, la póliza será efectiva desde el momento en que el buque "se desplace del puerto" hasta el momento en que se atraque o amarre en el lugar de destino. Como parece evidente, este segundo tipo de póliza se refiere al concepto de viaje que tradicionalmente se entiende como un único "ciclo operativo del barco" ${ }^{47}$ desde el puerto de salida hasta el puerto de destino ${ }^{48}$.

Por otro lado, las reglas que rigen la duración de las pólizas de mercancías se encuentra art. 532, párrafo 1, c. nav., el que establece, con carácter general, que la cobertura es eficaz desde el momento en que las mercancías salen de tierra para ser cargadas en el buque, hasta el momento en que son descargadas en el puerto de destino, siempre que, de conformidad con el párrafo segundo, la descarga de dichos bienes se produzca dentro de los treinta días siguientes a la llegada del buque al puerto ${ }^{49}$. De acuerdo con el art. 532, párrafo 3, c. nav., también se cubre el stock de mercancía en flotadores, en la medida en que esta actividad sea necesaria para la descarga y que este almacenamiento no dure más de quince días ${ }^{50}$. A pesar de la ley, cabe destacar el uso generalizado en los contratos de este tipo de cláusulas contractuales que tienen como objetivo ampliar la eficacia de la póliza para incluir también los riesgos que corre la mercancía en el transporte "de almacén a almacén"51.

\footnotetext{
45 Lefebvre D’Ovidio, Pescatore e Tullio. Manuale di diritto della navigazione, cit., p. 722; Ferrarini, Le assicurazioni marittime, cit., p. 330, quien considera que esta prórroga automática también se aplica en los casos en que el vencimiento del seguro de tiempo se produce cuando el buque se encuentra amarrado en un puerto de mero tránsito.

${ }^{46}$ De conformidad con el citado párrafo, si antes de la finalización de las operaciones de descarga "el buque embarca mercancías para un nuevo viaje, para lo cual el propio buque ha sido asegurado, el seguro anterior cesa con el inicio del nuevo embarque".

47 Cfr., Berlingieri. I diritti di garanzia sulla nave, Padova, 1965, p. 60 ss.

48 Sin embargo, sobre las dificultades interpretativas asociadas con la definición de "viaje", v. FERRARINI. Le assicurazioni marittime, cit., p. 333 lo que pone de relieve cómo, a pesar de la aparente sencillez de la noción, "no siempre es posible establecer cuáles son el puerto de salida y, sobre todo, el puerto de destino. Entonces, por ejemplo, si un barco sale para ir a un puerto a tomar una carga para llevarla a otro puerto, ¿el cruce de lastre constituye un viaje autónomo o es parte del viaje posterior? Entonces, nuevamente, ¿si el barco sale con diferentes cargas para varios puertos, solo hay un viaje o más viajes, y cuántos?”.

49 Este párrafo se entiende sin perjuicio de la hipótesis en la que el retraso en la descarga de la mercancía se deba a cuarentena o casos de fuerza mayor.

50 Lefebvre D’Ovidio, Pescatore e Tullio. Manuale di diritto della navigazione, cit., p. 723.

51 Sobre la cláusula “de almacén a almacén”, v. TuRCI, Le clausole «da magazzino a magazzino» nell'assicurazione marittima merci, in Assicurazioni, 1966, p. 95 ss.; CElle, La clausola «da magazzino a magazzino», in Dir. econ. ass., 1995, p. 599 ss.; PILlinini, Le coperture assicurative «da magazzino a magazzino» nel trasporto multimodale, in Dir. trasp., 1996, p. 449 ss.; BocCHESE, La clausola "da magazzino a magazzino" nell'esperienza assicurativa italiana, in Dir. trasp., 2005, p. 873 ss.; Moliterni, La clausola "da magazzino a magazzino" nell'assicurazione marittima delle merci e l'interpretazione del contratto, cit.,
} 
Merece especial atención la disciplina de la eficacia temporal de las llamadas pólizas de "viaje ya empezado", que son posibles tanto en el seguro de buques como en el de mercancías, las que encuentran su propia disciplina específica en el art. 531, párrafo 3 c. nav, el primero, y en el art. 532, párrafo 4, c. nav, el último. En ambos casos, el Código de Navegación establece que estas pólizas, concluidas cuando el viaje ya ha comenzado, entran en vigencia a partir de la hora indicada en la póliza o, si no se indica, a partir de la medianoche del día de la conclusión de la contrato ${ }^{52}$. Según parte de la doctrina, estos dos párrafos formalizarían la posibilidad de que los asegurados den efecto retroactivo a las pólizas marítimas ${ }^{53}$. Al dejar a las partes libres para indicar el momento a partir del cual la póliza debe liberar sus efectos, estas disposiciones permitirían a los asegurados anticipar la efectividad de la cobertura hasta un momento antes de la conclusión del contrato ${ }^{54}$. Sin embargo, no parece que la disposición mencionada pueda tener un carácter tan sistemático, ya que, en primer lugar, se refieren única y exclusivamente a pólizas de buques estipuladas por viaje y a las pólizas de mercancías, mientras que los contratos por tiempo. Para esta tipología de seguro, como se mencionó, el art. 530 c. nav., prevé la efectividad solo para el futuro (a partir de la medianoche del día de la celebración del contrato). En cambio, es precisamente la interpretación sistemática de los artículos 530, 531 y 532 c. nav., la que, por el contrario, abogaría a favor de la posición contraria a la posibilidad de otorgar contractualmente efectos retroactivos a los seguros marítimos. De hecho, todas estas disposiciones tienden a hacer que los efectos del seguro surtan efecto a partir de un tiempo posterior a su estipulación (desde la medianoche del día en que se estipula el contrato, desde el momento en que se inicia el viaje o se inicia la carga de la mercancía). Incluso en la disciplina específica que regula el seguro de bienes una vez iniciado el viaje, la ley demuestra cierta propensión a reafirmar la efectividad pro-futuro de estas pólizas identificando como criterio complementario que, a falta de indicación del tiempo por parte de los contratantes, dichos seguros se hacen efectivos a partir de la media noche del día de su estipulación ${ }^{55}$.

Se reitera, nuevamente, que la retroactividad de las pólizas tiene sentido práctico solo si a través de estos acuerdos se logra una conformación diferente de los riesgos asegurados. Como se mencionó anteriormente, las reglas contenidas en el art. $514 \mathrm{c}$. nav., dejan totalmente indiferente el hecho de hacer retroactivos los efectos de una póliza marítima ya que, por los hechos que sufren los bienes asegurados antes de la

p. 209 ss.; Rossi, La clausola “da magazzino a magazzino” estende la copertura assicurativa anche allo sbarco in amministrazione, al deposito e all'ispezione doganale, in Dir. trasp., 2012, p. 735 ss.

52 Destaca la necesidad, en tales casos, de investigar a fondo la voluntad de los contratantes para verificar cuáles son realmente las necesidades de seguro que pretenden lograr a través del contrato FerrariNI. Le assicurazioni marittime, cit., p. 331 (en relación con las de buques una vez iniciado el viaje) y p. 338 (en relación con las políticas de mercancías una vez iniciado el viaje).

53 Sobre este punto, véase ampliamente supra.

${ }^{54}$ Cfr., VernizzI. Il rischio putativo, cit., pp. 118 ss.

55 Incluso una interpretación literal de la regla, aunque no decisiva, parecería conducir aún más a la imposibilidad de pactar la retroactividad de las pólizas marítimas. Las disposiciones que rigen el seguro una vez iniciado el viaje, de hecho, dejan a las partes la posibilidad de identificar únicamente el momento a partir del cual los riesgos asegurados entran en cobertura. Si con esta disposición el legislador hubiera querido otorgar a las partes la posibilidad de retroactuar los efectos de la póliza, probablemente hubiera otorgado la posibilidad de identificar también el día a partir del cual deben correr los efectos del seguro. 
celebración del contrato, subordina la obligación de indemnización del asegurador al hecho objetivo de que en el momento de celebración del contrato no llegó la noticia del siniestro al lugar de celebración de la póliza o el lugar desde el cual se dio la orden de asegurar.

\section{CAMBIOS EN EL RIESGO ASEGURADO}

Una vez identificados los riesgos que pueden ser cubiertos por el seguro marítimo desde un punto de vista objetivo-y los límites dentro de los cuales es admisible esta transferencia de riesgo -, no se pueden descuidar los cambios significativos que puede sufrir la relación en función de hechos que determinen una variación de este elemento. El contrato de seguro, en general, describe una relación jurídica que perdura en el tiempo y que, como tal, está expuesta a contingencias. Estas, cuando afecten a la extensión del riesgo asegurado, requieren la adopción de precauciones reglamentarias específicas que, en las reglas generales del contrato de seguro contenidas en el Código Civil, son identificables en los artículos 1896-1898 ${ }^{56}$.

Cabe señalar de inmediato que el código de navegación prevé una disciplina específica solo en relación con la agravación del riesgo o su cambio que, debido a la relación que se crea entre la regulación del sector y el código civil ${ }^{57}$, lleva a afirmar que la hipótesis del cese del riesgo y su disminución durante el seguro quedan regidas también para el seguro marítimo, respectivamente, por el art. 1896 del Código Civil italiano y 1897 del Código Civil italiano ${ }^{58}$.

El Código de Navegación, por su parte, dicta una disciplina específica relacionada con la agravación del riesgo, que debe entenderse como un aumento en la intensidad del riesgo en comparación con la forma en que se asumió en el contrato, y su cambio, es decir la transformación del riesgo originalmente asumido- ${ }^{59}$. Esta disciplina en particular, identificada en artículos 522 s. C. nav., tiene como objetivo expreso ${ }^{60}$ derogar - en el contexto que nos interesa- la disciplina a que se refiere el art. 1898 del Código Civil italiano que, al establecer una regla vinculante ${ }^{61}$, impondría consecuencias legales

\footnotetext{
56 En general, sobre estas disposiciones, v. AngELICI. Aggravamento e altre modifiche del rischio, in Assicurazioni, 1985, I, pp. 551 ss; RossetTI. Il diritto delle assicurazioni, cit., pp. 795 ss.; GiamPaOlino. Le assicurazioni, cit., pp. 285 ss.

57 V. supra.

58 Esta es la opinión generalizada en la doctrina especializada. V., QuercI. Diritto della navigazione, cit., p. 640; Lefebvre D’Ovidio, Pescatore e Tullio. Manuale di diritto della navigazione, cit., p. 716.

59 Explica esa distinción, v. FerRARINI, Le assicurazioni marittime, cit., p. 168 s.

${ }^{60}$ Este es el significado del Informe al código de navegación n. 331.

${ }^{61}$ Cabe señalar, de hecho, que si bien el art. 1898 del Código Civil italiano está expresamente calificado como norma imperativa por el art. 1932 del Código Civil italiano, el art. 522 c. nav.-además de derogar la disposición primera antes citada-dicta una disciplina que puede ser renunciada por las partes dada la frase "salvo pacto en contrario" con la que se abre. V., sobre este punto, PERsico, Le assicurazioni marittime, cit., p. 228. Esto atestigua que, en términos de agravamiento y modificación del riesgo, las particularidades del sector marítimo exigen una mayor flexibilidad de la norma.
} 
que no están en línea con las particulares necesidades emergentes en el campo de los seguros marítimos.

Al querer identificar las especificidades que en este punto marcan la diferencia con respecto a la disciplina del código civil, conviene resaltar, en primer lugar, que en los términos del art. 522, párrafo 1 c. nav., el agravamiento del riesgo afecta la cobertura del seguro única y exclusivamente en el caso de que esté determinado por el "hecho del asegurado" "62. La agravación del riesgo, por lo tanto, no es relevante cualquiera que sea la causa, como lo es, sin embargo, en el art. 1898 del Código Civil italiano-pero solo cuando depende de la conducta del asegurado. Cuando dicha conducta determine una transformación o agravación del riesgo de tal manera que, si el nuevo estado de cosas hubiera estado presente desde el principio, el asegurador no hubiera contratado o hubiera concluido en diferentes condiciones, la aseguradora no responderá para el reclamo ${ }^{63}$.

Esta disciplina en particular se ve mitigada aún más por el segundo párrafo del art. 522 c. nav., en virtud del cual el asegurador sigue siendo obligado a mantener ileso al asegurado en todas las circunstancias en las que la agravación del riesgo esté determinada por actos realizados por el asegurado por el deber de "solidaridad humana", para proteger un interés también del asegurador o depende de un evento por el cual el mismo asegurador es responsable según el contrato ${ }^{64}$.

Finalmente, es necesario resaltar, siempre con el intento de identificar las diferencias entre la normativa sectorial y la de derecho común, cómo la obligación de indemnización permanece intacta incluso en todas las circunstancias en las que la agravación del riesgo producida por un hecho del asegurado, no haya influido concretamente en la ocurrencia del accidente o en el monto relativo de la indemnización. Este principio, dictado por la última parte del art. 522, párrafo 2 c. nav., según la doctrina especializada, marca una diferencia más con respecto a la disciplina a que se refiere el art. 1898 del Código Civil italiano según el cual la agravación del riesgo es siempre relevante independientemente de su impacto concreto sobre la causa del daño ${ }^{65}$.

\section{SIGUE: EL CAMBIO DE RUTA, VIAJE O BARCO}

Sobre las reglas generales relativas a la agravación o modificación del riesgo descritas en el art. 522 c. nav., la disposición del art. 523 c. nav. tipifica tres hipótesis peculiares de variaciones en el riesgo asegurado. En particular, si bien el cambio de ruta o viaje, en su disciplina unitaria, pueden calificarse como hechos idóneos para agravar el riesgo asegurado, el cambio de buque -que se produce en el caso de carga de bienes asegurados en un buque distinto al indicado en la póliza- materializaría un hecho que determina un cambio real en el riesgo.

${ }^{62}$ Cfr., PaSanisi, Aggravamento del rischio, in Dir. mar., 1958, p. 350 ss.

${ }^{63}$ Ferrarini. Le assicurazioni marittime, cit., p. 170 s.; Querci. Diritto della navigazione, cit., p. 641.

${ }^{64}$ Lefebvre D’Ovidio, Pescatore e Tullio. Manuale di diritto della navigazione, cit., p. $716 \mathrm{~s}$.

${ }^{65}$ FERRARINI. Le assicurazioni marittime, cit., p. 171. 
Llegando a la disciplina específica, mientras que el cambio de viaje suele significar el cambio del lugar de salida o llegada con respecto al indicado en el contrato ${ }^{66}$, con el cambio de ruta nos referimos a la hipótesis en la que, en la realización del viaje establecido, el barco sigue una ruta distinta a la esperada o habitual ${ }^{67}$. Estos casos reciben una disciplina conjunta en el art. 523, párrafos 1 y 2 c. nav. y, por lo tanto, son aplicables tanto a los seguros de buques estipulados para viajes como a los seguros de mercancías ${ }^{68}$. Estas variaciones, en primer lugar, son indiferentes a la relación de seguro en todas las circunstancias en las que se produzca un cambio forzado de ruta o viaje, incluyendo literalmente dentro de estas hipótesis también aquellos cambios impuestos por la necesidad de asistencia o rescate de barcos o aeronaves o de personas en peligro (artículo 522, párrafo 1 del Código Civil italiano). Según una parte de la doctrina, dado su tenor literal, la disposición en cuestión debe en todo caso referirse única y exclusivamente a las hipótesis del llamado "Rescate obligatorio"69. Por tanto, sólo en esta circunstancia quedaría la obligación de indemnizar cualquier siniestro que se produzca al asegurado. Una visión sistemática del primer párrafo del art. 523 c. nav., sin embargo, nos lleva a considerar más apropiado referir la disciplina dictada por la misma, como lo hace otra doctrina y obviamente salvo pacto en contrario, también a la hipótesis de rescate no obligatorio ${ }^{70}$. De hecho, conviene reiterar que el cambio de ruta o viaje materializan hipótesis tipificadas de agravación del riesgo y que, como tales, quedan en todo caso sujetos a la normativa general a que se refiere el mencionado art. 522 c. nav. Como se mencionó anteriormente, el segundo párrafo de la última regla mencionada requiere mantener intacta la obligación de indemnización del asegurador en todas las circunstancias en las que la agravación del riesgo venga determinada por el cumplimiento por parte del asegurado de las obligaciones solidarias. El alcance general de este principio permite sin duda incluir cualquier hipótesis de ayuda -tanto la obligatoria impuesta por la ley, como la opcional-, en el contexto del cumplimiento de un deber de solidaridad frente a quienes se encuentran en una situación actual de dificultad o peligro.

Mientras que en el caso de un cambio forzoso de ruta o viaje, el asegurador siempre será responsable del siniestro, en el caso de que dichos cambios sean imputables al hecho del asegurado, de conformidad con el art. 523, párrafo 2 c. nav., cualquier obligación de indemnización del asegurador se limita únicamente a los casos en que el accidente se produzca durante el viaje cubierto por el seguro y siempre que el cambio de ruta o viaje no haya afectado a la ocurrencia del mismo. Fuera de estos casos, por tanto, el asegurador no será responsable del hecho lesivo ${ }^{71}$.

\footnotetext{
${ }_{66}$ V., RAIMONDI. Spunti in merito agli effetti del cambiamento di viaggio sulla copertura assicurativa delle Institute Cargo Clauses, in Dir. trasp., 1994, pp. 475 ss.

${ }^{67}$ Querci. Diritto della navigazione, cit., p. 641.

${ }^{68}$ Cfr. Ferrarini. Le assicurazioni marittime, cit., p. 172 que cree que la regla es inaplicable para los contratos de seguro de buques estipulados en función del tiempo.

${ }^{69}$ En este sentido, Ferrarini. Le assicurazioni marittime, cit., p. 172, spec. nota n. 170. Se trataría por tanto de las hipótesis descritas, principalmente, en los artículos 485, 489 y 490 c. nav. Sobre este punto, véase QuercI, Diritto della navigazione, cit., pp. 556 ss.

70 Así, Lefebvre D’Ovidio, Pescatore e Tullio. Manuale di diritto della navigazione, cit., p. 717.

71 Querci. Diritto della navigazione, cit., p. 641.
} 
Por otro lado, el caso de cambio de buque regulado por el art. 523, párrafo $3 \mathrm{c}$. nav. que, al disciplinar las hipótesis de carga de mercancías en un buque distinto al señalado en el contrato, realiza una transformación del riesgo aplicable únicamente al seguro de mercancías. No es casualidad que el embarque de mercancías en un buque distinto al indicado en el contrato represente una hipótesis en la que el asegurador se libera de cualquier obligación de seguro en caso de accidente precisamente por la importancia de las características del buque en la evaluación de los riesgos relativos a las mercancías transportadas ${ }^{72}$.

En la hipótesis, poco frecuente en la práctica, del seguro de bienes in quovis -en el que, es decir, no se indica el buque en el que se cargarán los bienes-, cualquier obligación de indemnización del asegurador está sujeta a la comunicación oportuna por parte del asegurado del nombre del barco en el que se cargarán las mercancías. En ausencia de este requisito, el asegurador queda en cualquier caso liberado ${ }^{73}$.

\section{BIBLIOGRAFÍA}

Angelici. Aggravamento e altre modifiche del rischio, in Assicurazioni, 1985, I, p. 551 ss.

BALESTRA. Le assicurazioni marittime dei rischi di guerra, Milano, 1991

BERLINGIERI. I diritti di garanzia sulla nave, Padova, 1965.

BocCHESE. La clausola "da magazzino a magazzino" nell'esperienza assicurativa italiana, in Dir. trasp., 2005, p. 873 ss.

САРоTOSTI. Rischio putativo nell'assicurazione marittima di trasporto merci e nullità del contratto per avaria particolare preesistente all 'imbarco, in Foro it., 1961, I, c. 1346 ss.

Carbone, Celle e lopez De Gonzalo. Il diritto marittimo attraverso i casi e le clausole contrattuali, $5^{\text {a }}$ ed, Torino, 2015.

Celle. La clausola «da magazzino a magazzino», in Dir. econ. ass., 1995, p. 599 ss.

CORRIAS. Il problema dell'onerosità-gratuità nel contratto di assicurazione e nei contratti aleatori, in Riv. dir. civ., 2016, pp. 68 ss.

CORRIAS. L'assicurabilità dei fatti pregressi nelle assicurazioni della responsabilità civile, in Riv. it. Med. legale, 2019, pp. 1443 ss.

CORrias. La clausola "claims made" al vaglio delle Sezioni Unite: un'analisi a tutto campo, in Banca borsa tit. cred., 2018, II, p. 656 ss.

\footnotetext{
72 FerrarinI. Le assicurazioni marittime, cit., p. 169, según el cual "la carga de la mercancía asegurada en un buque distinto al señalado en el contrato, por crear una situación de riesgo completamente diferente a la asumida por el contrato, representa un cambio de riesgo, es decir, un riesgo ni siquiera cubierto por el contrato de seguro estipulado entre las partes".

${ }^{73}$ Querci. Diritto della navigazione, cit., p. 641; Lefebvre D’Ovidio, Pescatore e Tullio. Manuale di diritto della navigazione, cit., p. 717. Una excepción es la hipótesis de los contratos de seguro de mercancías que se transportan barcos de la línea.
} 
Corrias. Sulle peculiarità della disciplina del rischio nelle assicurazioni marittime, in Dir. trasp., 1995, pp. 51 ss.

De Gregorio, in De Gregorio e Fanelli. Il contratto di assicurazione, II, Milano, 1987, pp. 80 ss.

Di MARCo. La funzione del contratto di assicurazione marittima, in Assicurazioni, 2004, pp. 411 ss.

Donati. Trattato del diritto delle assicurazioni, vol. II, Milano, 1954.

Fanelli. Le assicurazioni, in Tratt. dir. civ. comm. Cicu e Messineo, Milano, 1973.

FERrari. Il problema dell'alea contrattuale, Napoli, 2001, p. 100.

FERRARINI. Le assicurazioni marittime, Milano, 1990.

Ferrarini. Polizze tipo italiane e clausolari inglesi di assicurazioni marittime, in Assicurazioni, 1981, I, pp.125 ss.

Gambino. L'assicurazione nella teoria dei contratti aleatori, Milano, 1964.

Giampaolino. Le assicurazioni, in Tratt. dir. comm. Buonocore, Torino, 2013.

GRIGOLI. In merito alla disciplina dell'assicurazione del rischio putativo, in Assicurazioni, 1978, pp. 620 ss.

Grigoli. Sulla delimitazione del rischio di guerra nell'assicurazione marittima, in Assicurazioni, 1973, II, 2, pp. 266 ss.

HeIss. Insurance Contracts in Rome I: Another Recent Failure of the European Legislature, in Yearbook of Private Int. Law, 2008, pp. 261 ss.

Lefebvre D’Ovidio, Pescatore e Tullio. Manuale di diritto della navigazione, 9a , Milano, 2000.

Manca. Studi di diritto della navigazione, Milano, 1961.

Mazzola. Sul concetto di interesse nel contratto di assicurazione: inquadramento teorico e profili applicativi, in Riv. dir. civ., 2019, pp. 1201 ss.

MOLITERNi. La clausola "da magazzino a magazzino" nell'assicurazione marittima delle merci e l'interpretazione del contratto, in Nuova giur. civ. comm., 2005, pp. 209 ss.

Mugavero. Ancora dubbi di legittimità sulle clausole "claims made": la parola di nuovo alle Sezioni Unite, in Giur. comm., 2018, pp. 501 ss.

PASANISI. Aggravamento del rischio, in Dir. mar., 1958, pp. 350 ss.

Persico. Le assicurazioni marittime, Genova, 1947.

Pillinini. Le coperture assicurative «da magazzino a magazzino» nel trasporto multimodale, in Dir. trasp., 1996, pp. 449 ss.

Piombino. L'impiego di clausolari stranieri nel contratto di assicurazione marittima: la soluzione adottata dalla nuova polizza corpi dell'ANIA, in Dir. mar., 1989, pp. 1216 ss.

Pretelli. Il regolamento comunitario sulla legge applicabile alle obbligazioni contrattuali (Roma I), in Eur. dir. priv., 2009, pp. 1083 ss.

QuERCI. Diritto della navigazione, Padova, 1989. 
RAIMONDI. Spunti in merito agli effetti del cambiamento di viaggio sulla copertura assicurativa delle Institute Cargo Clauses, in Dir. trasp., 1994, pp. 475 ss.

RicCARDelli. Lezione di diritto della navigazione: assicurazioni marittime, in Dir. trasp., 2017, pp. 940 ss.

Ricciardelli. Il vizio occulto nell'assicurazione della nave, Padova, 1970.

RighetTi. Trattato di diritto marittimo, III, Milano, 1994.

Rossetti. Il diritto della assicurazioni, I, Padova, 2011.

Rossi. La clausola “da magazzino a magazzino" estende la copertura assicurativa anche allo sbarco in amministrazione, al deposito e all 'ispezione doganale, in Dir. trasp., 2012, pp. 735 ss.

Salemi. Le assicurazioni marittime e trasporti, in CANDIAN e PACI (a cura di) Manuale di tecnica delle assicurazioni, Milano, 2002.

SiCCARDI. Le nuove Institute Time Clauses - Hull, in Dir. mar., 1984, pp. 975 ss.

TURCI. Le clausole «da magazzino a magazzino» nell'assicurazione marittima merci, in Assicurazioni, 1966, pp. 95 ss.

VERNIZZI. Il rischio putativo, Milano, 2010.

VERNIZZI. Rischio putativo ed assicurazioni retroattive tra diritto della navigazione e diritto comune, in Riv. dir. nav., 2016, pp. 219 ss.

Volpe Putzolu. Le assicurazioni. Produzione e distribuzione. Problemi giuridici, Bologna, 1992. 
